\title{
Lack of evidence for infectious SARS-CoV-2 in feces and sewage
}

\author{
Sandra Albert ${ }^{1}$ - Alba Ruíz ${ }^{2}$ Javier Pemán ${ }^{2}$ - Miguel Salavert ${ }^{2}$ Pilar Domingo-Calap ${ }^{1,3}$ (1)
}

Received: 11 May 2021 / Accepted: 1 July 2021 / Published online: 9 July 2021

(c) The Author(s), under exclusive licence to Springer-Verlag GmbH Germany, part of Springer Nature 2021

\begin{abstract}
The SARS-CoV-2 can be excreted in feces and can reach sewage systems. Determining the presence of infective viral particles in feces and sewage is necessary to take adequate control measures and to elucidate new routes of transmission. Here, we have developed a sample concentration methodology that allows us to maintain viral infectivity. Feces of COVID-19 patients and wastewater samples have been analyzed both by molecular methods and cell culture. Our results show no evidence of infective viral particles, suggesting that fecal-oral transmission is not a primary route. However, larger-scale efforts are needed, especially with the emergence of new viral variants.
\end{abstract}

Keywords SARS-CoV-2; COVID-19 · Viral infectious particles · Fecal-oral transmission $\cdot$ Wastewater

Severe acute respiratory syndrome coronavirus 2 (SARSCoV-2) has caused a pandemic affecting the entire world. Although the main transmission route is via droplets or aerosols [1], it has been shown that the virus can replicate in intestinal mucosa $[2,3]$, suggesting that viral excretion via feces could result in fecal-oral transmission of SARSCoV-2 [4-6]. Despite the efforts to isolate viral infectious particles from feces, little is known about the possibility of infecting new hosts. In a first approach, it is necessary to determine the proportion of patients excreting virus in stool by analyzing fecal samples and testing them by molecular methods to detect viral RNA. In a second step, positive samples should be used to inoculate target cells, to determine the presence of infectious viruses. Until date, only a few case reports have shown that viral infectious particles are found in stool, all in samples with high viral load [7-9]. In addition, sewage transmission has been proposed as the most plausible explanation for two independent outbreaks in China [10, 11]. However, further efforts should be done to assess the fecal-oral route in SARS-CoV-2, and provide data based on

Pilar Domingo-Calap

pilar.domingo@uv.es

1 Instituto de Biología Integrativa de Sistemas, Universitat de València-CSIC, Paterna, Spain

2 Instituto de Investigación Sanitaria La Fe, Hospital Universitari I Politècnic La Fe, Valencia, Spain

3 Department of Genetics, Universitat de València, Burjassot, Spain the presence of infectious viral particles confirming the new route of transmission [8], especially with the emergence of new variants.

Here, we propose the first attempt to evaluate the fecal excretion of SARS-CoV-2 in Spain, and the presence of viral infectious particles in feces of COVID-19 patients and sewage. This pilot study based on a small cohort has been approved by the ethics committee of the Instituto de Investigación Sanitaria La Fe (Valencia, Spain, registration number 2020-301-1). Eight COVID-19 patients admitted to the Hospital Universitari i Politècnic La Fe (Valencia, Spain) between June and December 2020 were enrolled. Five patients showed respiratory difficulties, and only three had gastrointestinal (GI) symptoms (Supplementary Table 1). In addition, SARS-CoV-2 positive wastewater samples at the time of high prevalence in the region under study were obtained from wastewater treatment plants of Valencia (Spain), belonging to the Empresa Pública de Saneamiento de Aguas Residuales (EPSAR, Generalitat Valenciana).

To proceed with the study, we first compared different concentration methods to maintain viral infectivity using a surrogate coronavirus, the transmissible gastroenteritis virus (TGEV). Three different methods were tested: flocculation, ultrafiltration, and high-speed centrifugation. Aluminum-driven flocculation was done following previous work (Randazzo et al., 2020). Ultrafiltration method was performed using Amicon tubes with $100 \mathrm{kDa}$ filters. Finally, high-speed centrifugation method was done by centrifugation at $80,000 \times \mathrm{g}, 3 \mathrm{~h} 30 \mathrm{~min}$, at $4{ }^{\circ} \mathrm{C}$. The concentration 
factor was obtained by RT-qPCR using in-house TGEV primers and probes, and by plaque assay infecting swine testicular (ST) cells. For maintaining infectivity, high-speed centrifugation was the best method (concentration factor: $31.29 \pm 6.49$ ), followed by ultrafiltration (concentration factor: $7.38 \pm 1.26)$. In contrast, infectivity was impaired by aluminum-driven flocculation (concentration factor: $0.02 \pm 0.02$ ). As for RNA detection, high-speed centrifugation obtained similar results to the aluminum-driven flocculation method (concentration factor: $14.98 \pm 0.91 \mathrm{vs.}$ $16.42 \pm 2.13$ ), whereas ultrafiltration was not suitable (concentration factor: $2.85 \pm 0.87$ ). For these reasons, the highspeed centrifugation method was chosen as the best in terms of concentration and maintenance of viral infectivity. This should be taken into account for future work, since depending on the concentration method, inactivation of viruses can result in false negative results [12].

Stool and urine samples from COVID-19 patients and sewage samples were concentrated by high-speed centrifugation. RNA extraction was performed with the Nucleospin RNA Virus Kit (Macherey-Nagel) and RT-qPCR was performed with the GoTaq ${ }^{\circledR}$ Probe 1-Step RT-qPCR System (Promega) using the U.S. Center for Disease Control N1 and N2 primers sets (2019-nCoV CDC EUA Kit, 1000rxn). For each RT-qPCR run, calibration curves were performed using the 2019-nCoV_N_Positive Control. The results showed that six of the eight patients had viral excretion in feces in at least one of the samples analyzed, ranging from $8.34 \times 10^{3}$ $\mathrm{gc} / \mathrm{L}$ to $6.74 \times 10^{6} \mathrm{gc} / \mathrm{L}$ (Supplementary Table 2). It is worth mentioning that one of the patients showed absence of viral shedding in feces despite having GI symptoms. The absence of viral shedding in this patient was not expected, and a possible explanation is that the sample was taken one month after the onset of the first symptoms, suggesting that perhaps earlier sampling might have different results. In parallel, urine samples were collected for patients to test for RNA detection. In our dataset, one patient showed viral shedding in urine in two consecutive samples, despite the low viral load found (ranging from $5.60 \times 10^{4} \mathrm{gc} / \mathrm{L}$ to $1.78 \times 10^{5} \mathrm{gc} / \mathrm{L}$ ). In addition, we wanted to sequence the RNA obtained in the feces to analyze the variability found in stool. Genomic sequencing was performed following the ARTIC protocol, and sequencing on the Illumina MiSeq platform [13]. Unfortunately, the viral loads obtained were too low for sequencing. We attempted to sequence the sample with highest viral load, but the results were insufficient for further analysis, since only $13.28 \%$ of the SARS-CoV-2 genome was covered, with a coverage of 2 .

Fecal and sewage samples with highest RNA detected by RT-qPCR were used to inoculate Vero E6 cells, as recommended cell models for SARS-CoV-2 infection [14]. Concentrated samples were resuspended in Dulbecco Modified Eagle Medium (DMEM) supplemented with $2 \%$ fetal bovine serum, non-essential amino acids, penicillin and streptomycin, and $25 \mathrm{mM}$ Hepes. All samples were analyzed undiluted and at 1/10 dilution to avoid potential sample inhibitors or cytotoxic molecules. Microscopy images were taken to detect cytopathic effects of cell monolayers. No cytopathic effect on Vero E6 cells was observed in any of the samples analyzed. In addition, supernatants were collected at $0 \mathrm{~h}$ post-infection (hpi), $48 \mathrm{hpi}$, and 6 days post-infection, and RT-qPCR was performed attempting to detect viral amplification. In all fecal samples tested, no SARS-CoV-2 RNA was detected (Supplementary Table 3). To determine the potential contribution of wastewater to SARS-CoV-2 transmission, eight sewage samples were tested and again, despite the high viral load obtained in the samples (ranging from $1.43 \times 10^{4} \mathrm{gc} / \mathrm{L}$ to $1.48 \times 10^{6} \mathrm{gc} / \mathrm{L}$ ) (Supplementary Table 4), we were unable to detect evidence of replication in our assays (Supplementary Table 5).

Our results are in agreement with those previously published on the detection of SARS-CoV-2 in the stool of COVID19 patients [15]. However, we were able to detect viral RNA in stool in 6/8 patients, being a high percentage compared to previously published results, and suggesting that the virus may be detected more frequently in stool than expected based on previously published results [16]. Furthermore, our results suggest that GI symptoms and the presence of SARS-CoV-2 in feces are not correlated, as only three patients had GI symptoms of which one did not show fecal shedding of virus, as previously shown [17]. This result could have implications for the use of anal swabs as recently suggested. However, the implementation of more sensitive detection methods associated with fecal samples could be an interesting solution to develop non-invasive easy-to-use, rapid tests, than can be performed by common users, avoiding nasopharyngeal swabs, since nasal self-swabbing can lead to inadequate sample collection resulting in false negative conclusions.

To unravel the fecal-oral route of SARS-CoV-2 transmission, fecal and sewage samples have been analyzed in cell culture for the presence of infectious viral particles. We would like to highlight that the concentration methods used are a determining factor, as some methods may impair viral infectivity as shown here. In our case, the lack of evidence of infectivity could be due to the low viral load, with high $\mathrm{Ct}$ values, in contrast with previously published results in which infectious coronavirus was found in a sample with a $\mathrm{Ct}$ value ranging 20-23 [7]. However, it should be mentioned that $\mathrm{Ct}$ values found in feces and wastewater are usually high. The fact that only a few cases of infectious particles in feces have been reported so far suggests that, although transmission through feces might exist, it should not be considered a major route. In agreement with our results, a recent study in China was unable to detect viable virus in hospital wastewater [18]. 
We strongly suggest increased effort to continue testing more patients, including asymptomatic ones, to get a broader view of viral shedding in feces. In addition, sewage sampled directly from sewers could be an interesting approach to detect possible infectious particles, avoiding the wastewater treatment plants, where viral particles could be inactivated due to external factors such as temperature, humidity, or chemical agents in the matrix, which may degrade or impact the envelope. Finally, improved detection methodologies using more sensitive and efficient techniques, both in molecular diagnostic and cell culture, could lead to different results. Therefore, further characterization of fecal and sewage samples is mandatory to unravel the possible fecal-oral route of SARS-CoV-2 transmission and will be an interesting topic for future research, especially with the emergence of new viral variants.

Supplementary Information The online version contains supplementary material available at https://doi.org/10.1007/s10096-021-04304-4.

Funding This research was funded by FONDO-COVID19 COV20/00210 by Instituto de Salud Carlos III and PTI Salud Global CSIC reference 202020E292 to P.D-C. P.D-C. was supported by a Ramón y Cajal contract from the Spanish Ministry of Science and Innovation, Call 2019.

Data availability The data that support the findings of this study are available from the corresponding author, upon reasonable request.

Code availability Not applicable.

\section{Declarations}

Ethics approval and consent to participate This study has been approved by the ethics committee of the Instituto de Investigación Sanitaria La Fe (Valencia, Spain), registration number 2020-3011. Informed consent was obtained from all individual participants included in the study.

Consent for publication The authors declare consent for publication.

Conflict of interest The authors declare no competing interests.

\section{References}

1. Zhang R, Li Y, Zhang AL, Wang Y, Molina MJ (2020) Identifying airborne transmission as the dominant route for the spread of COVID-19. PNAS 117:14857-14863

2. Xiao F, Tang M, Zheng X, Liu Y, Li X, Shan H (2020) Evidence for gastrointestinal infection of SARS-CoV-2. Gastroenterology 158:1831-1833

3. Lamers MM, Beumer J, van der Vaart J, Knoops K, Puschhof J, Breugem TI, Ravelli RBG, Paul van Schayck J, Mykytyn AZ, Duimel HQ, van Donselaar E, Riesebosch S, Kuijpers HJH, Schipper D, van de Wetering WJ, de Graaf M, Koopmans M, Cuppen E, Peters PJ, Haagmans BL, Clevers H (2020) SARS-CoV-2 productively infects human gut enterocytes. Science 369:50-54

4. Jones DL, Baluja MQ, Graham DW, Corbishley A, McDonald JE, Malham SK, Hillary LS, Connor TR, Gaze WH, Moura IB,
Wilcox MH, Farkas K (2020) Shedding of SARS-CoV-2 in feces and urine and its potential role in person-to-person transmission and the environment-based spread of COVID-19. Sci Total Environ 749:141364

5. Guo M, Tao W, Flavell RA, Zhu S (2021) Potential intestinal infection and faecal-oral transmission of SARS-CoV-2. Nat Rev Gastroenterol Hepatol 18:269-283

6. Elsamadony M, Fujii M, Miura T, Watanabe T (2021) Possible transmission of viruses from contaminated human feces and sewage: Implications for SARS-CoV-2. Sci Total Environ 755:142575

7. Xiao F, Sun J, Xu Y, Li F, Huang X, Li H, Zhao J, Huang J, Zhao, (2020) J Infectious SARS-CoV-2 in feces of patient with severe COVID-19. Emerg Infect Dis 26:1920-1922

8. Cuicchi D, Lazzarotto T, Poggioli G (2020) Fecal-oral transmission of SARS-CoV-2: review of laboratory-confirmed virus in gastrointestinal system. Int J Colorectal Dis 36:437-444

9. Jeong HW, Kim S-M, Kim H-S, Kim Y-I, Kim JH, Cho JY, Kim S, Kang H, Kim S-G, Park S-J, Kim E-H, Choi YK (2020) Viable SARS-CoV-2 in various specimens from COVID-19 patients. Clin Microbiol Infect 26:1520-1524

10. Kang M, Wei J, Yuan J, Guo J, Zhang Y, Hang J, Qu Y, Qian H, Zhuang Y, Chen X, Peng X, Shi T, Wang J, Wu J, Song T, He J, Li Y, Zhong N (2020) Probable evidence of fecal aerosol transmission of SARS-CoV-2 in a high-rise building. Ann Intern Med 173:974-980

11. Yuan J, Chen Z, Gong C, Liu H, Li B, Li K, Chen X, Xu C, Jing Q, Liu G, Qin P, Liu Y, Zhong Y, Huang L, Zhu B-P, Yang Z (2020) Sewage as a possible transmission vehicle during a Coronavirus Disease 2019 outbreak in a densely populated community: Guangzhou, China, April 2020. Clin Infect Dis ciaa1494. https://doi.org/ $10.1093 / \mathrm{cid} / \mathrm{ciaa} 1494$

12. Giacobbo A, Rodrigues MAS, Zoppas Ferreira J, Bernardes AM, de Pinho MN (2021) A critical review on SARS-CoV-2 infectivity in water and wastewater. What do we know? Sci Total Environ $774: 145721$

13. Quick J (2020) nCoV-2019 sequencing protocol. https://doi.org/ 10.17504/protocols.io.bbmuik6w

14. Ogando NS, Dalebout TJ, Zevenhoven-Dobbe JC, Limpens RWAL, van der Meer Y, Caly L, Druce J, de Vries JJC, Kikkert M, Bárcena M, Sidorov I, Snijder EJ (2020) SARS-coronavirus-2 replication in Vero E6 cells: replication kinetics, rapid adaptation and cytopathology. J Gen Virol 101:925-940

15. Amirian ES (2020) Potential fecal transmission of SARS-CoV-2: current evidence and implications for public health. Int J Infect Dis 95:363-370

16. Cheung KS, Hung IFN, Chan PPY, Lung KC, Tso E, Liu R, Ng YY, Chu MY, Chung TWH, Tam AR, Yip CCY, Leung K-H, Fung AY-F, Zhang RR, Lin Y, Cheng HM, Zhang AJX, To KKW, Chan K-H, Yuen K-Y, Leung WK (2020) Gastrointestinal manifestations of SARS-CoV-2 infection and virus load in fecal samples from a Hong Kong cohort: systematic review and meta-analysis. Gastroenterol 159:81-95

17. Parasa S, Desai M, Thoguluva Chandrasekar V, Patel HK, Kennedy KF, Roesch T, Spadaccini M, Colombo M, Gabbiadini R, Artifon ELA, Repici A, Sharma P (2020) Prevalence of gastrointestinal symptoms and fecal viral shedding in patients with Coronavirus Disease 2019. JAMA Netw Open 3:e2011335

18. Wang J, Feng H, Zhang S, Ni Z, Ni L, Chen Y, Zhuo L, Zhong Z, Qu T (2020) SARS-CoV-2 RNA detection of hospital isolation wards hygiene monitoring during the Coronavirus Disease 2019 outbreak in a Chinese hospital. Int J Infect Dis 94:103-106

Publisher's note Springer Nature remains neutral with regard to jurisdictional claims in published maps and institutional affiliations. 